FACULDADE DE CIÊNCIAS ECONÔMICAS DA UFRGS Análise
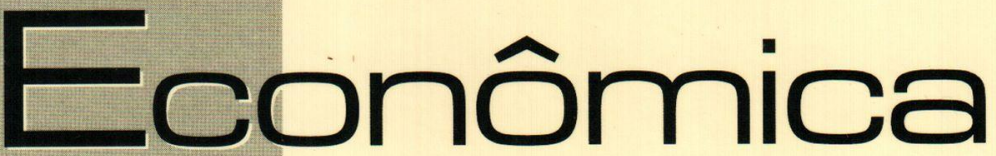

FLUTUACÃO CAMBIAL E METAS INFLACIONÁRIAS EM

ECONOMIAS EMERGENTES

OTAVIANO CANUTO E MárCIO HOLLAND

ATEORIA DE FORMACĆOO DE PRECOS E A TEORIA DOS PREÇOS DE

PRODUCAO

LOIZALBERTO ESTEVES

AECONOMIA EVOLUCIONISTA: UM CAPITULOSISTEMMICO DA

TEORIA ECONOMICA?

HUGOE. A. DA GAMACERQUEIRA

RELAÇ̃O INFLAÇÃO-PRODUTO NO BRASIL NO PERIODO PÓS-

REAL

MARCOSC. HOLANDA

DIVIIDA PÚBLICAMOBILLÍRIA FEDERAL BRASILEIRA: HISTÓRIA RECENTE E PERSPECTIVAS EXPLOSIVAS

ROGÉRIO MEURER E ROBERT WAYNE SAMOHYI

A CEPAL EA INTEGRACÃO REGIONAL LATINO-AMERICANA JACQUELINE A. HERNANDEZ HAFFNER

POPPER, HAYEK EA (IM)POSSIBILIDADE DE PREDIÇŌES ESPECIFICAS EM CIEENCIAS SOCIAIS

BRENA PAULA MAGNO FERNANDEZ

DINÂMICA RECENTE DO PROCESSO DE INCUBACG̃O DE

EMPRESAS DE BASE TECNOLOGICA NO BRASIL

EDUARDO GONÇALVES

TRIBUTACĀO COM SACRIF́CIO EQUUITATIVO: O CASO DO IMPOSTO DE RENDA PESSOA FISICA

STEFANO FLORISSI E EDUARDO PONTUAL RIBEIRO

A NOVATEORIA DO IMPÉRIOE AS VELHAS TEORIAS DO

IMPERIALISMO

GENTIL CORAZZA

Ô ABREALAS - A NOVA INSERÇÃO DO BRASIL NA ECONOMIA MUNDIAL

EMLLLANO LUIS KLEN

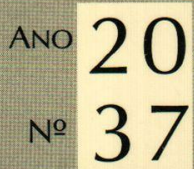

Março, 2002 
UNIVERSIDADE FEDERAL DO RIO GRANDE DO SUL

Reitora: Profa. Wrana Maria Panizzi

FACULDADE DE CIÊNCIAS ECONÔMICAS

Diretor: Prof. Pedro Cézar Dutra Fonseca

CENTRO DE. ESTUDOS E PESQUISAS ECONÔMICAS

Diretor: Prof. Gentil Corazza

DEPARTAMENTO DE CIÊNCIAS ECONÔMICAS

Chefe: Prof. Luiz Alberto Oliveira Ribeiro de Miranda

DAPARTAMENTO DE CIÊNCIAS CONTÁBEIS E ATUARIAIS

Chefe: João Marcos Leão da Rocha

CURSO DE PÓS-GRADUAÇÃO EM ECONOMIA

Coordenador: Prof. Eduardo Pontual Ribeiro

CURSO DE PÓS-GRADUAÇÃO EM ECONOMIA RURAL

Coordenador: Prof Jalcione Almeida

CONSELHO EDITORIAL: Carlos G. A. Mielitz Netto (UFRGS), Eduardo A. Maldonado Filho (UFRGS), Eduardo P. Ribeiro (UFRGS), Eleutério F. S. Prado (USP), Eugênio Lagemann (UFRGS), Fernando Cardim de Carvalho (UFRJ), Fernando Ferrari Filho (UFRGS), Fernando de Holanda Barbosa (FGV/RJ), Flávio Vasconcellos Comim (UFRGS), Gentil Corazza (UFRGS), Giácomo Balbinotto Neto (UFRGS), Gustavo Franco (PUC/RJ), Jan A. Kregel (Università di Bologna e John Hopkins University), João Rogério Sanson (UFSC), Joaquim Pinto de Andrade (UnB), Jorge Paulo Araújo (UFRGS), Juan H. Moldau (USP), Marcelo S. Portugal (UFRGS), Maria Alice L.ahorgue (UFRGS), Paul Davidson (University of Tennessee), Paulo Dabdab Waquil (UFRGS), Pedro Cézar Dutra Fonseca (UFRGS), Philip Arestis (South Bank University), Roberto C. Moraes (UFRGS), Ronald Otto Hillbrecht (UFRGS), Sabino da Silva Porto Jr. (UFRGS), Stefano Florissi (UFRGS), Werner Baer (Univ, of Illinois at Urbana-Champaign).

COMISSÃO EDITORIAL: Eduardo Augusto Maldonado Filho, Fernando Ferrari Filho, Gentil Corazza, Marcelo Savino Portugal, Paulo Dabdab Waquil; Roberto Camps Moraes.

EDITOR: Fernando Ferrari Filho

EDITOR ADJUNTO: Gentil Corrazza

SECRETÁRIA: Vanessa Hoffmann de Quadros

REVISÃO DE TEXTOS: Vanete Ricacheski

FUNDADOR: Prof. Antônio Carlos Santos Rosa

Os materiais publicados na revista Análise Econômica são da exclusiva responsabilidade dos autores. É permitida a reprodução total ou parcial dos trabalhos, desde que seja citada a fonte. Aceita-se permuta com revistas congêneres. Aceitam-se, também, livros para divulgação, elaboração de resenhas e recensões. Toda correspondência, material para publicação (vide normas na terceira capa), assinaturas e permutas devem ser dirigidos ao seguinte destinatário:

PROF FERNANDO FERRARI FILHO

Revista Análise Econômica - Av. João Pessoa, 52 CEP 90040-000 PORTO ALEGRE - RS, BRASIL Telefones: (051) 316-3348 e 316-3440 - Fax: (051) 316-3990

E-mail: rae@vortex ufrgs br

Análise Econômica

Ano 20, n 37 , março, 2002 - Porto Alegre

Faculdade de Ciências Econômicas, UFRGS, 2000

Periodicidade semestral, março e setembro.

1. Teoria Econômica - Desenvolvimento Regional Economia Agrícola - Pesquisa Teórica e Aplicada -

Periódicos. I. Brasil.

Faculdade de Ciências Econômicas,

Universidade Federal do Rio Grande do Sul.

CDD 330.05

CDU 33 (81) (05) 


\title{
Relação inflação-produto no Brasil no período Pós-Real
}

\author{
Marcos C. Holanda*
}

Resumo: O artigo estima curvas de oferta e demanda agregada para a economia brasileira no período que segue o Plano Real. As estimativas são feitas de forma desagregada entre os principais setores da economia e exploram a importância da variável crédito na determinação do produto e da inflação.

Palavras-chave: Inflação, Produto, Demanda e Oferta Agregadas.

Abstract: The article estimates aggregate supply and demand functions for Brazilian economy following the Real Plan. The estimates consider the main sector of economy and explore the importance of domestic credit in determining the country output and inflation.

Key words: Inflation, Output, Aggregate Demand and Supply. Código JEL: E5, C2.

\section{Introdução}

Em junho de 1999, o Brasil adotou como estratégia de política monetária um regime de metas de inflação. Em tal regime, a política monetária opera com algum grau de discricionismo que é restringido pela meta de inflação a ser alcançada ${ }^{1}$.

Como bem salienta Svensson (1997), um regime de metas de inflação é, na verdade, um regime de metas de previsāo de inflação. Isto é, dada a reconhecida defasagem com que os juros impactam a atividade econômica e, conseqüentemente a inflação, o Banco Central é obrigado a operar a taxa de juros não em função da inflação do período, mas sim em função de uma inflação esperada.

A operacionalizaçāo do regime requer, dessa forma, a construção e estimação de modelos estruturais da economia que permitam a simulação de impactos dos juros período na inflação futura.

\footnotetext{
- Professor Titular CAEN - UFC. Somos gratos ao apoio financeiro do CNPq através de bolsa de Produtividade em Pesquisa - PQ.

${ }^{1}$ Sobre o assunto, ver Bogdanski, Tombini e Werlang (1999) e Bernarke (1999).
} 
A maneira mais simples de modelar uma economia é representála a partir das curvas de oferta e demanda agregadas. A demanda agregada é representada pela curva IS, que relaciona a atividade econômica à taxa de juros. A oferta agregada é representada pela curva de Phillips, onde a inflação é função do nível de atividade econômica.

Apesar da simplicidade, a representação da economia a partir das curvas IS e de Phillips é bastante popular entre os Bancos Centrais que operam em regimes de meta de inflação. Além disso, como chama a atenção Svensson (1997), elas possuem a atratividade de: a) usar taxa de juros de curto prazo como instrumento de política e não agregados monetários; b) a atividade econômica ser especificada em função de um hiato de produto, e não de taxas de crescimento do mesmo; c) trabalhar com Curvas de Phillips que incorporam expectativas autoregressivas compatíveis com a hipótese de taxa natural de produção; d) apresentar bom ajuste aos dados empíricos de vários países.

O objetivo do presente artigo é estimar curvas IS e de Phillips, dentro do modelo proposto por Svensson (1997), para a economia brasileira no período Pós-Real.

Tais estimações já são, na verdade, em diferentes graus de complexidade, freqüentes na literatura empírica nacional (Bogdanski, Tombini e Werlang (1999), Freitas e Muinhos (1999), Gonçalves (2000)).

Em relação a tal literatura, o artigo inova em dois pontos principais. Primeiro, estima as curvas IS e de Phillips de forma desagregada entre os principais setores da economia (indústria, serviços e agricultura). Segundo, explora de forma detalhada a importância da variável crédito na determinação do produto e da inflação.

A primeira seção apresenta o modelo básico a ser estimado com suas respectivas desagregações. A seção dois mostra os principais resultados encontrados. A seção três conclui o trabalho.

\section{O modelo}

O modelo é composto de duas equaçōes:

$$
\begin{aligned}
& \pi_{t}=c+\sum_{i=1}^{3} \alpha_{i} \pi_{t-i}+\sum_{i=0}^{2} \delta_{i} h_{t-i}+\sum_{i=0}^{2} \theta_{i} \Delta C N_{t-i}+\varepsilon_{t} \\
& h_{t}=c+\sum_{i=1}^{3} \beta_{i} h_{t-i}-\sum_{i=0}^{2} \lambda_{i}\left(\mathrm{l}_{t-i}-\pi_{t-i}\right)+\sum_{t=0}^{2} \phi_{i} C R E_{t-i}+\sum_{t=o}^{2} \omega_{i} C R_{t-i}+\sum_{i=0}^{2} \mu_{i} N F S P_{t-i}+\eta_{t}
\end{aligned}
$$


Na equação (1), Curva de Phillips, $\pi$ é a taxa de inflação medida pelo IPCA-IBGE, h é o hiato de produto definido pela diferença entre o PIB atual e o PIB potencial, e $\Delta \mathrm{CN}$ é a variação da taxa de câmbio nominal Real/Dólar.

A Curva de Phillips definida em (1) assume a hipótese de expectativas auto-regressivas ou extrapolativas. Tal hipótese, apesar de menos atrativa do ponto de vista teórico, tem-se mostrado não inferior, do ponto de vista empírico, à hipótese de expectativas forward-looking. Além disso, como menciona Svensson (1997), ela é mais compativel com períodos iniciais de implantação de um regime de metas onde os agentes ainda não conhecem bem a nova regra de política a ser adotada.

A inclusão da variável $\Delta \mathrm{CN}$ procura captar o impacto na inflação de repasses de aumento de custos associados à variação cambial. Ela é particularmente importante para o período de estimação, considerado que é caracterizado por fortes oscilaçōes do câmbió2.

Para o cálculo da variável hiato do produto, o produto potencial é definido em função do filtro de Hodrik-Prescott (H-P). O uso do filtro H-P, mesmo estando sujeito a críticas, é muito freqüente na literatura em função de seus resultados empíricos satisfatórios ${ }^{3}$.

Na equação (2), Curva IS, $\left(i_{t}, \pi_{i}\right)$ são os juros reais definidos pelos juros nominais over-selic deflacionados pela inflação do IPCA, CRE é uma variável representando a oferta de crédito real na economia, CR é a taxa de câmbio real e NFSP é a necessidade de financiamento do setor público.

A inclusão da variável CRE é justificada pela hipótese de que, em uma economia que opera com restrição de crédito como a brasileira, o crédito, assim como os juros são importantes no processo de transmissão monetária ${ }^{4}$. As variáveis de câmbio real e necessidade de financiamento do setro público procuram captar pressões de demanda advindas do setor externo e da política fiscal, respectivamente.

A estimação do modelo é feita por OLS, e as variáveis têm freqüência trimestral, cobrindo o período que vai do primeiro trimestre de 1995 até o quarto trimestre de 2000.

\footnotetext{
${ }^{2}$ A variável $\triangle \mathrm{CN}$ pode também ser interpretada como uma proxy para expectativas futuras da inflação, caso em que a equação estimada incorporaria expectativas backward e forward-looking. ${ }^{3}$ Para uma discussáo sobre metodologias alternativas de cálculo do PIB potencial, ver Guay (1999). ${ }^{4}$ O papel do crédito no processo de transmissão monetária é discutido em Brunner e Meltzer(1998) e em Bernarke e Blinder(1998).
} 
As equaçōes são estimadas para diferentes conjuntos de hiato de produto e oferta de crédito. Isto é, no caso da variável hiato de produto, são estimadas Curvas IS para o hiato de produto geral (HPIBG), industrial (HPIBI), de serviços (HPIBS) e agrícola (HPIBA), bem como suas respectivas Curvas de Phillips. Além disso, para cada hiato de produto setorial são testados os impactos de diferentes modalidades de oferta de crédito. Tais modalidades são crédito industrial, crédito comercial, crédito rural, crédito privado total, crédito a pessoa física, e crédito do governo federal, dentro da classificação das estatísticas de crédito geradas pelo Banco Central do Brasil. Ao todo, são estimadas um total de 48 equações.

A idéia básica é procurar identificar qual setor da economia é mais sensível a juros e a crédito, bem como a sensibilidade da inflação a hiatos de produto em tais setores.

\section{As estimações}

Como primeiro passo para as estimações das diferentes equações mencionadas, são testadas as estacionaridades das variáveis consideradas. A tabela 1 resume os resultados dos testes ADF implementado.

Tabela 1: Testes de Estacionaridade das Séries

\begin{tabular}{c|c|c}
\multicolumn{3}{|c}{ Testes ADF } \\
\hline Variável & ADF & $\begin{array}{c}\text { Valor crítico } \\
(5 \%)\end{array}$ \\
\hline IPCA & $-3,53$ & $-3,61$ \\
\hline HPIBG & $-5,61$ & $-2,93$ \\
\hline HPIBI & $-5,47$ & $-2,93$ \\
\hline HPIBS & $-5,72$ & $-2,93$ \\
\hline HPIBA & $-7,12$ & $-2,93$ \\
\hline JR & $-2,98$ & $-2,99$ \\
\hline DCN & $-5,42$ & $-3,61$ \\
\hline$M$ & $-3,14$ & $-2,99$ \\
\hline CPT & $-0,56$ & $-3,61$ \\
\hline DCPT & $-2,78$ & $-3,03$ \\
\hline CPF & $-1,41$ & $-3,61$ \\
\hline DCPF & $-4,04$ & $-3,67$ \\
\hline Cl & $-0,85$ & $-2,99$ \\
\hline DCI & $-2,25$ & $-3,67$ \\
\hline$C C$ & $-1,43$ & $-3,61$ \\
\hline$D C C$ & $-3,56$ & $-3,03$ \\
\hline CR & $-1,04$ & $-3,61$ \\
\hline DCR & $-4,09$ & $-3,67$ \\
\hline
\end{tabular}


Apesar da baixa potência do teste $\mathrm{ADF}$ e do tamanho limitado das séries, todas as variáveis, com exceção da variável oferta de crédito, se mostram estacionárias em nível. A série de oferta de crédito só se mostra estacionária em primeira diferença. Para efeito de estimação do modelo, o uso da variável crédito em primeira diferença faz todo sentido, já que é de se esperar que a pressão de demanda seja definida pelo fluxo e não pelo estoque de crédito.

As equações são estimadas com uma estrutura de defasagem mais ampla possível, tendo em vista as limitaçōes de tamanho das séries. No geral, defasagens acima de dois trimestres não se mostraram significativas. A tabela 2 resume os melhores resultados obtidos nas estimações das Curvas IS.

Tabela 2: Curvas IS Estimadas

\begin{tabular}{|c|c|c|c|c|c|c|c|c|c|c|}
\hline Varióvel dependente & \multicolumn{5}{|c|}{ HPIBG } & \multicolumn{5}{|c|}{$\mathrm{HPIB|}$} \\
\hline Variável explicafiva & 163 & 2224 & 17]1 & 1.453 & & 1630 & 2726 & 1695 & 1.291 & 3,12 \\
\hline C & 1,681 & $(2,247)$ & $0,744)$ & 0 & $\begin{array}{l}20,65) \\
\end{array}$ & $\begin{array}{l}10,163] \\
\end{array}$ & (1,85i) & 0,20 & 10,815 & $(2,03)$ \\
\hline $\mathrm{H}(-\mathrm{l})$ & $\begin{array}{l}0,123 \\
10,715)\end{array}$ & $\begin{array}{l}0,0885 \\
(0,4871)\end{array}$ & $\begin{array}{l}0,0781 \\
0,428 \mid\end{array}$ & $\begin{array}{l}0,228 \\
01,259)\end{array}$ & $\begin{array}{l}0.144 \\
0851\end{array}$ & $\begin{array}{l}0,389 \\
(2,525)\end{array}$ & $\begin{array}{r}0,369 \\
{[2,211}\end{array}$ & $\begin{array}{l}0,320 \\
1,961\end{array}$ & $\begin{array}{l}0,520 \\
(3,15)\end{array}$ & $\begin{array}{l}0,424 \\
0,591 \\
\end{array}$ \\
\hline$H(-2)$ & $\begin{array}{r}-0,35 \\
-2,05) \\
\end{array}$ & $\begin{array}{r}-0,281 \\
-1,6231\end{array}$ & $\begin{array}{l}-0,345 \\
(1,1,985)\end{array}$ & $\begin{array}{l}0,340 \\
i-1,854)\end{array}$ & $\begin{array}{l}-0,390 \\
(-2,27)\end{array}$ & $\begin{array}{r}-0,475 \\
-3,088) \\
\end{array}$ & $\begin{array}{l}0.366 \\
-2,191 \\
\end{array}$ & $\begin{array}{l}-0,450 \\
-2,901\end{array}$ & $\begin{array}{l}-0,53 \\
1-3,091 \\
1.30\end{array}$ & $\begin{array}{r}0,455 \\
1.2761\end{array}$ \\
\hline $\mathbb{R}(-1)$ & $\begin{array}{l}-0,012 \\
-1,0181\end{array}$ & $0,0,0804$ & $\begin{array}{l}-0,0127 \\
(-1,604)\end{array}$ & $0.0,0111$ & $\begin{array}{r}-0,0207 \\
(-2,64)\end{array}$ & $\begin{array}{l}0.0129 \\
0.1,13211 \\
\end{array}$ & $\begin{array}{l}0,0271 \\
1-2,18\}\end{array}$ & 0,0124 & 0,0097 & $\begin{array}{l}0,025 \\
(-2,07)\end{array}$ \\
\hline DCREPT & $\begin{array}{l}10,069 \\
{[2,195)}\end{array}$ & & & & & $\begin{array}{l}1759 \\
12,5111\end{array}$ & & & & \\
\hline DCREPF & & $\begin{array}{l}54,603 \\
01,9801 \\
\end{array}$ & & & & & $\begin{array}{l}87,98 \\
(2,144\end{array}$ & & & \\
\hline DCREI & & & $\begin{array}{l}18,097 \\
{[2,030)}\end{array}$ & & & & & $\begin{array}{l}33,23 \\
{[2,63)}\end{array}$ & & \\
\hline DCREC & & & & $\begin{array}{l}7,217 \\
0,4031\end{array}$ & & & & & $\begin{array}{l}33,90 \\
0,871\end{array}$ & \\
\hline DCRER & & & & & $\begin{array}{l}137,91 \\
{[2,371} \\
\end{array}$ & & & & & $\begin{array}{l}173,67 \\
0,9791\end{array}$ \\
\hline$R^{2}$ & 0,44 & 0,421 & 0,426 & 0,367 & & 0,587 & 0,539 & 0,58 & 0,517 & 0,526 \\
\hline S.E & 2,5 & 2,55 & 2,53 & 2,66 & & 3,57 & 3,77 & 3,59 & 3,86 & 3,82 \\
\hline D.W & 2,089 & 1,946 & 1,99 & 2,174 & & 1,57 & 1,45 & 1,44 & 1,58 & 1,196 \\
\hline Variável dependente & \multirow{2}{*}{\multicolumn{5}{|c|}{ HPIBS }} & \multirow{2}{*}{\multicolumn{5}{|c|}{ HPIBA }} \\
\hline Variável explicativa & & & & & & & & & & \\
\hline C & $\begin{array}{r}0,459 \\
10,915 ! \\
\end{array}$ & $\begin{array}{r}0,673 \\
0,3621 \\
\end{array}$ & $\begin{array}{l}0,47 \\
10,861 \\
\end{array}$ & $\begin{array}{l}0,474 \\
10,9611 \\
\end{array}$ & $\begin{array}{l}0,761 \\
0.4611 \\
\end{array}$ & $\begin{array}{l}6,9377 \\
11,6171\end{array}$ & $\begin{array}{l}8,081 \\
0,891\end{array}$ & $\begin{array}{l}6,511 \\
0,44)\end{array}$ & $\begin{array}{l}7,13 \\
0,651 \\
\end{array}$ & $\begin{array}{l}9.27 \\
12,181\end{array}$ \\
\hline $\mathrm{H}(-1)$ & $\begin{array}{l}0,319 \\
0,7561\end{array}$ & $\begin{array}{l}0,278 \\
\pi, 501\end{array}$ & $\begin{array}{l}0,358 \\
8,851 \\
\end{array}$ & $\begin{array}{l}0,287 \\
0,5741 \\
\end{array}$ & $\begin{array}{l}0,341 \\
0,841 \\
\end{array}$ & $\begin{array}{l}-0,366 \\
(-2,0048)\end{array}$ & $\begin{array}{l}0,422 \\
-2,24\}\end{array}$ & $\begin{array}{l}0,341 \\
(1,87)\end{array}$ & $\begin{array}{l}0,359 \\
(1,94)\end{array}$ & $\begin{array}{l}0,392 \\
1-2,2751\end{array}$ \\
\hline$H(-2)$ & $\begin{array}{r}0,3,32 \\
0,1,78) \\
\end{array}$ & $\begin{array}{l}-0,28 \\
(-1,501\end{array}$ & $\begin{array}{l}0,320 \\
1.1,541\end{array}$ & 0,317 & $\begin{array}{l}0,33 \\
0,1,71) \\
(-1)\end{array}$ & $\begin{array}{l}-0,608 \\
-3,527)\end{array}$ & $\begin{array}{l}-0,646 \\
(-3,72) \\
\end{array}$ & $\begin{array}{l}0.0,581 \\
i \cdot 3,25)\end{array}$ & $\begin{array}{r}0,608 \\
1.3,441 \\
\end{array}$ & $\begin{array}{l}-0,687 \\
(-4,00)\end{array}$ \\
\hline$J R(-1)$ & $\begin{array}{r}-0,00502 \\
i-1,260\} \\
\end{array}$ & $\begin{array}{l}0,0078 \\
(-1,94\} \\
\end{array}$ & $\begin{array}{l}0,0051 \\
-1,146)\end{array}$ & $\begin{array}{l}-0,0049 \\
(1,26)\end{array}$ & $\begin{array}{c}-0,00757 \\
(-1,83)\end{array}$ & $\begin{array}{l}0,0573 \\
(-1,677)\end{array}$ & $\begin{array}{l}0,0725 \\
(-2,047)\end{array}$ & $\begin{array}{l}0,0,035 \\
(1,476)\end{array}$ & 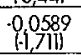 & $\begin{array}{l}0,0,0761 \\
{[-2,25]}\end{array}$ \\
\hline DCREPT & $\begin{array}{r}3,51 \\
0,491 \\
\end{array}$ & & & & & $\begin{array}{l}10,313 \\
10,5401 \\
\end{array}$ & & & & \\
\hline DCREPF & & $\begin{array}{l}20,67 \\
0,521\end{array}$ & & & & & $\begin{array}{l}123,65 \\
0,051 \\
\end{array}$ & & & \\
\hline DCREI & & & & $\begin{array}{l}7,01 \\
0,04) \\
104\end{array}$ & & & & & $\begin{array}{l}5,598 \\
10,1541 \\
\end{array}$ & \\
\hline DCREC & & & $\begin{array}{l}4,02 \\
10,621 \\
\end{array}$ & & & & & $\begin{array}{l}24,41 \\
10,481)\end{array}$ & & \\
\hline DCRER & & & & & $\begin{array}{l}37,169 \\
11,211\end{array}$ & & & & & $\begin{array}{r}384,08 \\
0,581 \\
\end{array}$ \\
\hline$R^{2}$ & 0,34 & 0,35 & 0,28 & 0,36 & 0,32 & 0,48 & 0,50 & 0,48 & 0,47 & 0,53 \\
\hline S.E & 1,28 & 1,28 & 1,27 & 1,27 & 1,31 & 10,739 & 10,51 & 10,75 & 10,81 & 10,17 \\
\hline D.W & 1,83 & 1,76 & 1,81 & 1,81 & 1,53 & 2,73 & 2,74 & 2,78 & 2,78 & 2,50 \\
\hline
\end{tabular}


O efeito dos juros no hiato de produto acontece com uma defasagem de um trimestre, sendo, como esperado, negativo. $O$ efeito dos juros na atividade econômica mostra-se bem mais rápido do que nos casos de economias desenvolvidas, onde tal efeito acontece entre três e quatro trimestres.

Os resultados mostram que a variável crédito também é importante na determinação da atividade econômica. Como os sinais dos coeficientes das variáveis juros e crédito são contrários, deve-se sempre considerá-los em conjunto na análise dos efeitos da política monetária no nível de atividade da economia. Isto é, aumento de juros quando acompanhados por aumentos de oferta de crédito podem representar uma política monetária expansionista e não contracionista.

Enquanto os juros afetam a economia com defasagem de um trimestre, o efeito da expansão de crédito é imediato.

O PIB agrícola é o que se mostra mais sensível e o PIB de serviços menos sensível a juros. O PIB industrial fica na posição intermediária.

Em relação a crédito, o PIB industrial é o que se mostra mais sensivel, em especial no que se refere a crédito a pessoa física. Cabe salientar que o crédito rural também tem um efeito importante no PIB industrial. Esse resultado indica que uma maior atividade agrícola induz uma maior atividade industrial, e não o contrário.

No geral, o crédito comercial é aquele com menor nível de significância na explicação da atividade econômica.

As variáveis câmbio real e necessidade de financiamento do setor público, apesar de apresentarem os sinais esperados, negativo e positivo, respectivamente, mostraram baixo nível de significância.

As estimações para as Curvas de Phillips são apresentadas na tabela 3 .

O impacto do hiato do PIB na inflação acontece com defasagem de dois trimestres. Como o impacto dos juros no PIB acontece com defasagem de um trimestre, a política monetária afeta a inflação com uma defasagem de três trimestres.

A inflação mostra-se mais sensível ao PIB de serviços e, em seguida, ao PIB agrícola. O PIB de serviços, por sua vez, mostrou-se pouco sensível a juros.

A maior sensibilidade da inflação aos PIB de serviços e agrícola indicaria uma menor capacidade desses setores, em relação ao setor industrial, de responder a pressões de demanda com aumentos de produção. 
Tabela 3: Curvas de Phillips Estimadas

\begin{tabular}{c|c|c|c|c|c|c|c|c|c|c|c|c}
$\begin{array}{c}\text { Variável } \\
\text { dependente }\end{array}$ \\
$\begin{array}{c}\text { Varível } \\
\text { explicativa }\end{array}$
\end{tabular}

A variável variação do câmbio nominal mostra-se mais importante na determinação da inflação do que a variável atividade econômica. Uma possível explicação seria que, no período considerado, as pressōes de demanda agregada foram acomodadas, predominantemente, via déficit nas contas externas e não via inflação.

Como variável explicativa adicional para a inflação, utilizamos as importações. O sinal negativo de seu coeficiente sugere que, liquidamente, as importações tendem a pressionar para baixo a inflação. Isto é, o efeito disciplinador dos preços internos de uma maior oferta de bens no mercado doméstico predomina sobre o efeito de maiores custos dos importados.

Por outro lado, o sinal negativo da variável importações e o sinal positivo da variável câmbio nominal podem ser interpretados como um indicador de que o efeito maior do câmbio na inflação aconteceu via preços administrados como energia, telecomunicações, petróleo, etc. 


\section{Conclusões}

A operacionalização de um regime de metas de inflação requer da parte do Banco Central um esforço contínuo de estimação da interação entre oferta e demanda agregadas da economia. É a partir de um melhor conhecimento dessa interação que ele pode aperfeiçoar sua performance na condução da política monetária.

O presente artigo estima curvas de oferta e demanda agregadas para a economia brasileira no período pós-Plano Real. As estimaçōes são feitas em níveis desagregados de PIB e oferta de crédito setoriais.

Por um lado, os resultados mostram que a política monetária afeta a inflação com uma defasagem de três trimestres e que ela deve ser operacionalizada considerando não apenas os juros, mas também a oferta de crédito. Por outro, eles mostram também que, pelo menos para o período considerado, o câmbio é mais importante do que o nível de atividade econômica na definição de inflação. Nesse caso, a política monetária deveria ter como parâmetro principal o primeiro e nāo o segundo.

O desafio é como compatibilizar esse resultado empírico com a hipótese de câmbio flutuante que um regime de metas de inflação considera.

\section{Referências bibliográficas}

BERNARKE, B. e BLINDER, A. (1998), "Credit, Money, and Aggregate Demand?", AEA papers and proceedings, p. 435-439.

BERNARKE, LOUBACH, MISHKIN e POSEN (1999), Inflation Targeting, Princeton University Press.

BOGDANSKI, J., TOMBINI, A. e WERLANG, S. (1999), Implementing Inflation Targeting in Brazil, Banco Central do Brasil, texto para discussão $n^{\circ} 1$.

BRUNNER, K. e MELTZER, A (1998), Money or Credit in the Monetary Transmission Process, AEA papers and proceedings, p. 446-451.

FREITAS, P. e MUINHOS, M. (1999), A Simple Model for Inflation Targeting in Brazil, Banco Central do Brasil, texto para discussão n ${ }^{\circ} 18$.

GONÇALVES, C. (2000), Metas de Inflação e Mecanismos de Transmissäo de Politica Monetária: o Caso Brasileiro, IPE/USP, texto para discussão n 14/ 2000. GUAY, A. e ST-AMART, P. (1999), A Survey of Alternative Methodologies for Estimating Potencial Output and the Output Gap, Journal of Macroeconomics, v. $21, n^{\circ} 3$, p. 557-595. 
HODRICK, R.J. and E.C. PRESCOTT. 1997. "Postwar U.S. Business Cycles: Na Empirical Investigation”. Journal of Money, Credit, and Banking 29(1):1-16.

SVENSSON, L. e RUDEBUSCH, G. (1997), Policy Rules for Inflation Targeting, NBER Conference on Monetary Rules, mimeo. 